\title{
Cytotoxic Components Against Human Oral Squamous Cell Carcinoma Isolated from Andrographis paniculata
}

\author{
RYUICHIRO SUZUKI ${ }^{1}$, YASUAKI MATSUSHIMA ${ }^{1}$, NORIYUKI OKUDAIRA ${ }^{2}$, \\ HIROSHI SAKAGAMI ${ }^{2}$ and YOSHIAKI SHIRATAKI ${ }^{1}$ \\ ${ }^{1}$ Faculty of Pharmaceutical Sciences, Josai University, Sakado, Japan; \\ ${ }^{2}$ Division of Pharmacology, Meikai University School of Dentistry, Sakado, Japan
}

\begin{abstract}
Background: The 5-year survival rate of patients with oral cancer has remained approximately $50 \%$ during the past 30 years, possibly due to the poor tumor selectivity of conventional anticancer drugs. This prompted us to search for new candidates for anticancer drugs that have higher cytotoxicity and tumor selectivity. Materials and Methods: Dried leaves of Andrographis paniculata were supplied from a market in Shanghai. The methanolic fraction of A. paniculata was further fractionated to identify cytotoxic principles by spectroscopic analysis and comparison with literature values. Viable cell number was determined by the 3-[4,5-dimethylthiazol-2-yl]-2,5-diphenyltetrazolium bromide method, and tumor specificity was calculated by relative cytotoxicity against oral squamous cell carcinoma cell lines compared to that against normal oral cells. Apoptosis induction was detected by cleaved poly (ADP-ribose) polymerase and caspase-3 on western blot analysis. Results: Major cytotoxicity in the methanol extract of a leaf of $A$. paniculata was recovered by partitioning with EtOAc, followed by silica gel chromatography. Further purification with reversed-phase high-performance liquid chromatography led to isolation of four known cytotoxic compounds, 14-deoxyandrographolide, andrographolide, neoandrographolide and deoxyandrographiside. Among them, andrographolide had the greatest cytotoxicity and tumor specificity, also inducing caspase-3 activation of HSC2 oral squamous cell carcinoma cells. Conclusion: The present study identified andrographolide as a major antitumor principle in the methanolic extract of leaves of $A$. paniculata.
\end{abstract}

Correspondence to: Professor Yoshiaki Shirataki, Faculty of Pharmaceutical Sciences, Josai University, 1-1 Keyakidai, Sakado, Saitama 350-0295, Japan. Tel: +81 492717053, Fax: +81 492717984, e-mail: shiratak@josai.ac.jp

Key Words: Andrographis paniculata, cytotoxicity, human oral squamous cell carcinoma.
Head and neck cancer is the sixth most common malignancy, compromising approximately $6 \%$ of all cancer cases (1), and mostly comprised of heterogeneous populations of oral squamous cell carcinomas $(1,2)$. At the early stage of oral cancer progression, surgery and radiation are the most popular choice of antitumor treatment (2), but they may cause disfiguring and psychological trauma in patients. Therefore, the development of new treatment strategies and the early diagnosis of oral cancer are of great importance (3). We have initiated a series of studies to search for new natural substances for the treatment of oral cancer, using an in vitro evaluation system with human oral squamous cell carcinoma cell lines (HSC-2, HSC-3 and HSC-4) and human normal oral cells (gingival fibroblast, HGF; periodontal ligament fibroblast, HPLF; pulp cell, HPC). Since the methanolic extract of Andrographis paniculata was found to have relatively higher cytotoxic activity, we isolated and identified the cytotoxic principles present in this extract, and also investigated the possibility of apoptosis induction of HSC-2 cells by compound isolated from this extract.

\section{Materials and Methods}

Materials. The following chemicals and reagents were obtained from the indicated companies: Dulbecco's modified Eagle's medium (DMEM; Gibco BRL, Grand Island, NY, USA); fetal bovine serum (FBS; JRH Bioscience, Lenexa, KS, USA); 5-fluorouracil (5-FU; Kyowa, Tokyo, Japan); doxorubicin hydrochloride (Wako Pure Chem. Ind., Osaka, Japan); dimethyl sulfoxide (DMSO; Wako Pure Chem. Ind.); DMSO- $d_{6}, \mathrm{CD}_{3} \mathrm{OD}$ (Cambridge Isotope Laboratories, Inc., MA, USA) and pyridine- $d_{5}$ (ACROS organics, Geel, Belgium); silica gel (Fuji Silysia Chemical LTD., Aichi, Japan) and silica gel plate (Merck KGaA, Damstadt, Germany). Protease and phosphatase inhibitors were purchased from Roche Diagnostics (Tokyo, Japan).

Antibodies against cleaved caspase-3 (Cell Signaling Technology Inc., Beverly, Maryland), poly (ADP-ribose) polymerase (PARP) (Cell Signaling Technology Inc') and glyceraldehyde 3-phosphate dehydrogenase (GAPDH; Trevigen, Gaithersburg, MD, USA) were used as primary antibodies. As secondary antibodies, we used $\alpha$ rabbit IgG (DAKO Japan) antibodies which were conjugated with horseradish peroxidase. 
General experimental procedures. Nuclear magnetic resonance (NMR) spectra were measured on a $400 \mathrm{MHz}$ Agilent-400MR-vnmrs 400 spectrometer (Agilent Technologies, Santa Clara, CA, USA) at room temperature using standard pulse sequences. Electron ionization-mass spectra (EI-MS) were measured with a JEOL JMN 700 spectrometer (JEOL Ltd., Tokyo, Japan). Electrospray ionization (ESI)-MS was also measured with an API4000-QTRP (AB SCIEX, CA, USA). Open column chromatography was performed on silica gel. Highperformance liquid chromatography (HPLC) was performed with Senshu Pak ODS column $(4.6 \mathrm{~mm}$ i.d. $\times 150 \mathrm{~mm}$ for analysis, $10 \mathrm{~mm}$ i.d. $\times 250 \mathrm{~mm}$ for preparation; Senshu Scientific Co., Ltd., Tokyo, Japan). AQUASIL SP100 (10 mm i.d. × 250 mm; Senshu Scientific Co., Ltd.) was also used as preparative column for HPLC purification.

Plant materials. The dried leaves of A. paniculata were supplied from a market in Shanghai. The specimen was proven and identified by Dr. Y. Shirataki and voucher specimens (\#20140521) were also deposited in the Department of Pharmacognosy and Natural Medicines of Josai University.

Preparation of methanolic extract. Methanolic extract of leaves of A. paniculata $(3 \mathrm{~kg})$ was prepared by extraction with $\mathrm{MeOH}$ under reflux for $3 \mathrm{~h}$. This extract was completely dried in vacuo to give the corresponding dried extract (146 g).

Isolation of components from methanolic extract. The methanolic extract was suspended in water, then extracted with EtOAc and $n$ $\mathrm{BuOH}$, successively. Each soluble portion was evaporated in vacuo to give EtOAc (57.2 g) and $n$ - $\mathrm{BuOH}$ (43.1 g) fractions, respectively. The EtOAc fraction was chromatographed on a silica gel column by step-wise elution with a $\mathrm{CHCl}_{3}-\mathrm{MeOH}$ mixture $\left(\mathrm{CHCl}_{3}\right.$ : $\mathrm{MeOH}=100: 1 \rightarrow 1: 1 \rightarrow 1: 3)$ to give three fractions (Fr 1-3). Fr $2(31.2$ g) was further purified with silica gel open-column chromatography eluting with $\mathrm{CHCl}_{3}-\mathrm{MeOH}$ mixture $\left(\mathrm{CHCl}_{3}: \mathrm{MeOH}=100: 1 \rightarrow 50: 1 \rightarrow\right.$ $30: 1 \rightarrow 15: 1 \rightarrow 7: 1 \rightarrow 3: 1 \rightarrow 0: 1)$ to provide seven further fractions (Fr. 2-1-2-7). Fr. 2-1 (0.92 g) and Fr. 2-2 (1.4 g) combined with Fr. 2-3 (1.0 g) were purified by reversed-phase HPLC, eluting with a $\mathrm{MeCN}-\mathrm{H}_{2} \mathrm{O}$ mixture, to afford compound $\mathbf{1}(87.8 \mathrm{mg})$ and $\mathbf{2}(123.8$ $\mathrm{mg})$ respectively. Fr. 2-4 (1.5 g) was subjected to reversed-phase HPLC using aqueous MeCN as solvent system to give compound 3 (21.4 mg). Fr. 2-5 (2.8 g) was rechromatographed on silica gel open column eluting with $\mathrm{CHCl}_{3}-\mathrm{MeOH}$ mixture $\left(\mathrm{CHCl}_{3}: \mathrm{MeOH}=6: 1 \rightarrow\right.$ $3: 1 \rightarrow 0: 1$ ) to provide three fractions (Fr. 2-5-1 - 2-5-3). Fr. 2-5-1 was purified with HPLC (AQUASIL SP100, $10 \mathrm{~mm}$ i.d. $\times 250 \mathrm{~mm}$, $\mathrm{CHCl}_{3}-\mathrm{MeOH}$ mixture) to give compound 4 (54.3 mg).

Cell culture. Human oral squamous cell carcinoma cell lines (HSC2, HSC-3, HSC-4) were purchased from Riken Cell Bank (Tsukuba, Ibaraki, Japan). Normal human oral cells, HGF, HPLF and HPC were prepared from periodontal tissues, according to the guideline of the Intramural Ethic Committee (No. A0808), after obtaining informed consent from the 12-year-old patient at the Meikai University Hospital (4). Since normal oral cells have a limited lifespan of 43-47 population doubling levels (PDL) (4), they were used at 8-15 PDL in this study. All the cells were cultured in DMEM supplemented with $10 \%$ heat-inactivated FBS, $100 \mathrm{U} / \mathrm{ml}$ penicillin $\mathrm{G}$ and $100 \mu \mathrm{g} / \mathrm{ml}$ streptomycin sulfate. The normal cells were detached by $0.25 \%$ trypsin- $0.025 \%$ EDTA-2Na in phosphate-buffered saline without $\mathrm{Mg}^{2+}$ and $\mathrm{Ca}^{2+}[\mathrm{PBS}(-)]$ and subcultured at a 1:4 split ratio once a week, with a medium change between the subcultures.
Assay for cytotoxic activity. The cells were seeded $\left(2.5 \times 10^{3}\right.$ cells/well, $0.1 \mathrm{ml} /$ well) in 96-microwell plates (Becton Dickinson and Company, Franklin Lakes, NJ, USA) and incubated for $48 \mathrm{~h}$ to allow cell attachment. Near-confluent cells were treated for $48 \mathrm{~h}$ with different concentrations of the test compounds in fresh medium. The relative viable cell number of adherent cells was then determined by the 3-[4,5-dimethylthiazol-2-yl]-2,5-diphenyltetrazolium bromide (MTT) method (5). In brief, control and treated cells were incubated for $4 \mathrm{~h}$ with $0.2 \mathrm{mg} / \mathrm{ml}$ of MTT in the culture medium. After removing the medium, the reaction product, formazan, was extracted with DMSO and the absorbance (corresponding to the relative viable cell number) was measured at $540 \mathrm{~nm}$ by a microplate reader (Multiskan Bichromatic Labsystems, Helsinki, Finland). The $50 \%$ cytotoxic concentration $\left(\mathrm{CC}_{50}\right)$ was determined from the dose-response curve. The tumor-specificity (TS) was calculated by the following equation: $\mathrm{TS}=$ mean $\mathrm{CC}_{50}$ (normal cells)/mean $\mathrm{CC}_{50}$ (all tumor cell lines).

Western blott analysis. The cells were washed with PBS and resuspended in $50 \mathrm{mM}$ Tris- $\mathrm{HCl}(\mathrm{pH} 7.6), 150 \mathrm{mM} \mathrm{NaCl}, 1 \mathrm{mM}$ EDTA, $0.1 \%$ SDS, $0.5 \%$ deoxycholic acid, $1 \%$ NP-40, and protease inhibitors (RIPA buffer). After ultrasonication using Bioruptor (UCD-250, Cosmo Bio Tokyo, Japan) for $12.5 \mathrm{~min}(10 \mathrm{~s}$ on, $20 \mathrm{~s}$ off) at the middle level of output $(250 \mathrm{~W})$ at $4{ }^{\circ} \mathrm{C}$. The soluble cellular extracts were recovered after centrifugation for $10 \mathrm{~min}$ at $16,000 \times g$. The protein concentration of each sample was determined using BCA Protein Assay Reagent Kit (Thermo Fisher Scientific Waltham, MA, USA), and cell extracts were subjected to western blot analysis. The blots were probed with primary antibody against PARP or caspase- 3 followed by a horseradish peroxidaseconjugated secondary antibody. The immune complexes were visualized using Pierce Western Blotting Substrate Plus (Thermo Fisher Scientific). Western blot results were documented and quantified using ImageQuant LAS 500 (GE Healthcare Japan, Tokyo, Japan).

Statistical treatment. Experimental values are expressed as the mean \pm standard deviation (SD). Statistical analysis was performed by using Student's $t$-test. A $p$-value of less than 0.05 was considered to be significant.

\section{Results}

Isolation of active components. During the search for candidate natural products for the treatment of oral cancer, methanolic extract of $A$. paniculata was found to show slightly higher cytotoxic activity against oral squamous cell carcinoma (HSC-2, HSC-3 and HSC-4) than human oral normal cells (HGF, HPLF, HPC), with an approximate TS of 3 (Table I). When the $\mathrm{MeOH}$ extract was partitioned with successive organic solvents, the EtOAc-soluble fraction had the strongest cytotoxicity. Therefore, the EtOAc-soluble fraction was further purified to yield three fractions. Among of them, Fr. 2 exhibited the strongest activity. Thus, Fr. 2 was further chromatographed to give corresponding fractions (Frs. 2-1, 2-2, 2-3, 2-4, 2.5, 2.6 and 2-7). The cytotoxicity and tumor-specificity of each fraction are shown in Table I. 
Table I. Cytotoxicity of silica gel chromatography fractions prepared from the methanol extract of Andrographis paniculata. The cells were incubated for $48 \mathrm{~h}$ without or with test compounds and the 50\% cytotoxic concentration $\left(C C_{50}\right)$ value was determined by 3-[4,5-dimethylthiazol-2-yl]-2,5diphenyltetrazolium bromide method. Each value represents the mean of triplicate assays.

\begin{tabular}{|c|c|c|c|c|c|c|c|c|c|}
\hline \multicolumn{10}{|c|}{$\mathrm{CC}_{50}(\mu \mathrm{g} / \mathrm{ml})$} \\
\hline & \multicolumn{4}{|c|}{ Human oral squamous cell carcinoma cell lines } & \multicolumn{4}{|c|}{ Human normal oral cells } & \multirow[b]{2}{*}{ TS } \\
\hline & HSC-2 & HSC-3 & HSC-4 & Mean & HGF & HPLF & HPC & Mean & \\
\hline $\mathrm{MeOH}$ extr. & $34 \pm 15$ & $26 \pm 2$ & $21 \pm 7$ & 28 & $67 \pm 10$ & $83 \pm 26$ & $72 \pm 8$ & 74 & 3 \\
\hline Fr. 2-1 & $36 \pm 3$ & $33 \pm 2$ & $29 \pm 1$ & 32 & $193 \pm 3$ & $161 \pm 5$ & $89 \pm 2$ & 148 & 5 \\
\hline Fr. 2-2 & $7 \pm 1$ & $5 \pm 2$ & $7 \pm 1$ & 5 & $41 \pm 1$ & $29 \pm 0$ & $12 \pm 1$ & 27 & 5 \\
\hline Fr. $2-3$ & $11 \pm 1$ & $6 \pm 0$ & $7 \pm 1$ & 7 & $48 \pm 3$ & $29 \pm 1$ & $13 \pm 5$ & 30 & 4 \\
\hline Fr. 2-4 & $46 \pm 5$ & $39 \pm 4$ & $15 \pm 2$ & 36 & $98 \pm 6$ & $165 \pm 5$ & $>250$ & $>171$ & 5 \\
\hline Fr. 2-5 & $99 \pm 6$ & $182 \pm 29$ & $97 \pm 10$ & 112 & $>250$ & $>250$ & $243 \pm 77$ & $>248$ & 2 \\
\hline Fr. 2-6 & $131 \pm 9$ & $213 \pm 7$ & $139 \pm 18$ & 145 & $>250$ & $>250$ & $>250$ & $>250$ & 2 \\
\hline Fr. 2-7 & $76 \pm 2$ & $134 \pm 5$ & $80 \pm 10$ & 84 & $>250$ & $>250$ & $208 \pm 6$ & $>236$ & 3 \\
\hline $5-\mathrm{FU}$ & $<8$ & 36 & 24 & 23 & $>1000$ & $>1000$ & $>1000$ & $>1000$ & $>43$ \\
\hline DXR & $<0.06$ & 0.21 & 0.08 & 0.12 & $>7.5$ & $>7.5$ & $>7.5$ & $>7.5$ & $>63$ \\
\hline
\end{tabular}

TS, Tumor-specificity index; HGF, human gingival fibroblast; HPC, human pulp cells; HPLF, human periodontal ligament fibroblast; DXR, doxorubicin; 5-FU, 5-fluorouracil.

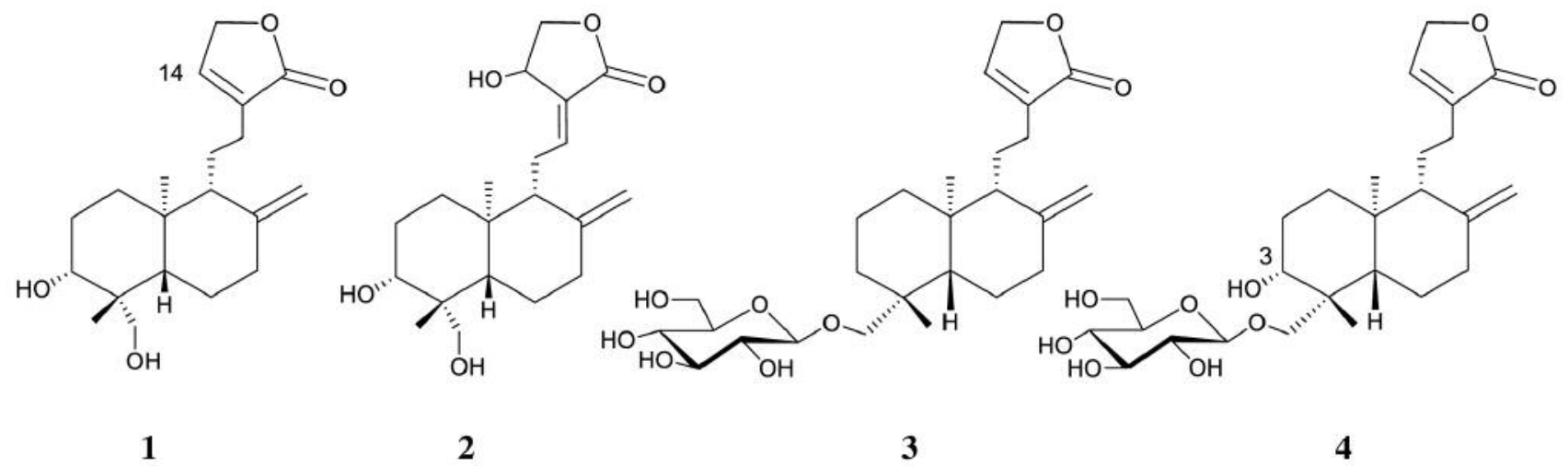

Figure 1. Structures of major cytotoxic compounds from the methanolic extract of Andorgraphis paniculata: 14-deoxyandrographolide [1], andrographolide [2], neoandrographolide [3] and deoxyandrographiside [4].

Identification of isolated compounds 1-4. Utilizing HPLC, major cytotoxic substances in Frs. 2-1, 2-2/2-3, 2-4 and 2-5 were isolated as compounds $\mathbf{1}, \mathbf{2}, \mathbf{3}$ and $\mathbf{4}$, respectively. On the basis of spectroscopic analysis including NMR, MS and comparison with those described in the respective literature, these compounds were identified as 14-deoxyandrographolide [1] (6), andrographolide [2] (6), neoandrographolide [3] (7) and deoxyandrographiside [4] (8), respectively (Figure 1).

Cytotoxic activities of isolated compounds. The cytotoxicities of isolated compounds are shown in Table II. Among four isolated compounds, andrographolide had the highest cytotoxicity against human oral squamous tumor cells (mean $\left.\mathrm{CC}_{50}=8.0 \mu \mathrm{M}\right)$ and the greatest tumor specificity $(\mathrm{TS}=9)$ (Table II). Neoandrographolide exhibited very weak cytotoxicity against both human tumor cells and normal cells. Deoxyandrographiside exhibited 2- to 3-fold lower cytotoxicity than deoxyandrographolide (Table II). Our preliminary study demonstrated that treatment of HSC-2 cells with 16 or $32 \mu \mathrm{M}$ andrographolide induced the cleavage of PARP and capase-3, suggesting the activation of caspase3 (Figure 2). 
Table II. Cytotoxicity of isolated compounds 1-4. The cells were incubated for 48 h without or with test compounds and the 50\% cytotoxic concentration $\left(\mathrm{CC}_{50}\right)$ value was determined by 3-[4,5-dimethylthiazol-2-yl]-2,5-diphenyltetrazolium bromide method. Each value represents the mean of triplicate assays.

\begin{tabular}{|c|c|c|c|c|c|c|c|c|c|}
\hline & \multicolumn{7}{|c|}{$\mathrm{CC}_{50}(\mu \mathrm{M})$} & \multirow[b]{3}{*}{ Mean } & \multirow[b]{3}{*}{ TS } \\
\hline & \multicolumn{4}{|c|}{ Human oral squamous cell carcinoma cell lines } & \multicolumn{3}{|c|}{ Human normal oral cells } & & \\
\hline & HSC-2 & HSC-3 & HSC-4 & Mean & HGF & HPLF & HPC & & \\
\hline 1 & $259 \pm 8.5$ & $267 \pm 35.5$ & $327 \pm 38.5$ & 204 & $381 \pm 14.5$ & $357 \pm 4.5$ & $380 \pm 0.5$ & 373 & 1 \\
\hline 2 & $8 \pm 1.0$ & $7 \pm 1.5$ & $10 \pm 4.0$ & 8.0 & $151 \pm 6.0$ & $25 \pm 0.5$ & $32 \pm 11.5$ & 69 & 9 \\
\hline 3 & $>1,000$ & $>1,000$ & $>1,000$ & $>1,000$ & $>1,000$ & $>1,000$ & $>1,000$ & $>1,000$ & $><1$ \\
\hline 4 & $509 \pm 19.5$ & $489 \pm 1.0$ & $729 \pm 7.0$ & 576 & $>1000$ & $943 \pm 57.5$ & $>1000$ & $>981$ & $>2$ \\
\hline DXR & 0.39 & 0.45 & 0.31 & 0.38 & 7.5 & 7.5 & 6.0 & 7.0 & 18 \\
\hline
\end{tabular}

TS, Tumor specificity index; HGF, human gingival fibroblast; HPC, human pulp cells; HPLF, human periodontal ligament fibroblast; DXR, doxorubicin.

\section{Discussion}

The present study identified andrographolide as the major antitumor principle in the methanolic extract of the leaves of A. paniculata. During the purification of andrographolide, the TS value increased from 3 (methanolic extract), to 4-5 (EtOAc partition, followed by silica gel chromatography) to 9 (final purification step with reversed phase HPLC) (Tables I and II). For the determination of TS, we used human malignant (HSC-2, HSC-3, HSC-4) and non-malignant (HGF, HPLF, HPC) cells as target cells, since the TS value determined by the present method well correlated with their in vivo antitumor activity, regardless of different cell types (either epithelial or mesenchymal) (9). For example, 7ethyl-10-hydroxycamptothecin (SN-38), the active metabolite of irinotecan used as clinical antitumor medicine, had a TS value of 808 by this assay method (10).

We next considered the structure-activity relationship of isolated components. The cytotoxicity of andrographolide against human oral squamous cell carcinoma cell lines $\left(\mathrm{CC}_{50}=8 \mu \mathrm{M}\right)$ was higher than that of 14-deoxyandrographolide $\left(\mathrm{CC}_{50}=204 \mu \mathrm{M}\right)$. The differences in chemical structure between these compounds are the position of the double bond on the tetrahydrofuran (endo- or exo-) and existence of a hydroxyl group connected at C-14. Thus, the hydroxyl group connected at $\mathrm{C}-14$ and the position of the olefin bond are important for cytotoxicity against tumor cells. Furthermore, glycosylation of these diterpenoids significantly reduced their cytotoxic activity against tumor cells, as shown by the comparison of neoandrographolide $\left(\mathrm{CC}_{50}>1,000 \mu \mathrm{M}\right)$ and deoxyandrographiside $(576 \mu \mathrm{M})$. Based on comparison of the structures of these glycosides, the existence of a hydroxyl group at the $\mathrm{C}-3$ position is essential for their cytotoxicity.

The present study demonstrated the possible induction of apoptosis in HSC-2 cells by andrographolide. Although
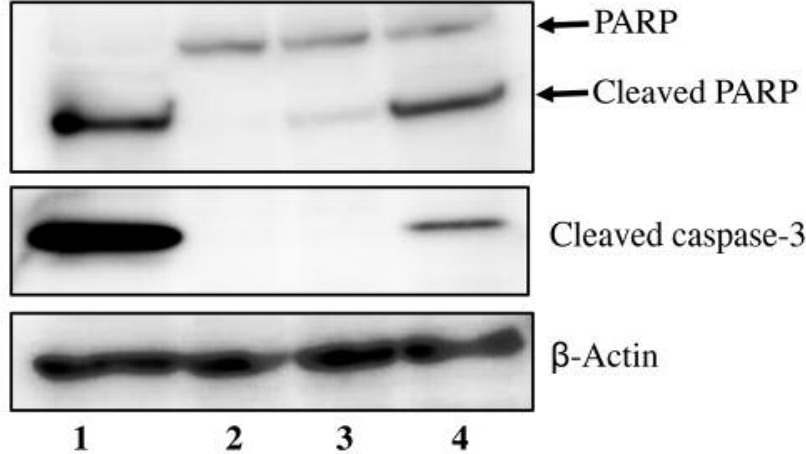

Figure 2. Induction of apotosis by andrographolide [2] in HSC-2 cells. HSC-2 cells were treated for $24 \mathrm{~h}$ with actinomycin $D\left(\begin{array}{ll}1 \mu M \\ \text { ) }\end{array}\right.$ (apoptosis-positive control) (lane 1), vehicle (negative control) (lane 2), andrographolide [2] (16,32 $\mu \mathrm{M})$ (lane 3, 4), and then processed for western blot analysis. PARP: Poly (ADP-ribose) polymerase.

induction of apoptosis by andrographolide via nuclear factorkappa B inhibition has been reported (11-14), very few studies have dealt with its tumor specificity (15). It remains to be investigated why this compound exhibits tumorselective cytotoxicity.

\section{Conflicts of Interest}

The Authors confirm that there are no conflicts of interest associated with this publication and there has been no significant financial support for this work that could have influenced its outcome.

\section{Acknowledgements}

This work was supported in part by Grant-in-Aid for Challenging Exploratory Research from The Ministry of Education, Culture, Sports, Science and Technology, research funds from Meikai University School of Dentistry (Sakagami H. 25670897). 


\section{References}

1 Irani S: miRNAs signature in head and neck squamous cell carcinoma metastasis. J Dent Shiraz Univ Med Sci 17: 71-83, 2016.

2 Economopoulou P, Perisanidis C, Giotakis I E and Psyrri A: The emerging role of immunotherapy in head and neck squamous cell carcinoma (HNSCC): anti-tumor immunity and clinical applications. Ann Transl Med 4: 173-185, 2016.

3 Ozi MJ, Suffredini BI, Paciencia M, Frana AS and Dib LL: In vitro cytotoxic effects of Brazilian plant extracts on squamous cell carcinoma of the oral cavity. Braz Oral Res 25: 519-525, 2011.

4 Kantoh K, Ono M, Nakamura Y, Nakamura Y, Hashimoto K, Sakagami $\mathrm{H}$ and Wakabayashi $\mathrm{H}$ : Hormetic and anti-radiation effects of tropolone-related compounds. In Vivo 24: 843-852, 2010

5 Masuda Y, Ueda J, Tamura M, Sakagami H, Tomomura M, Tomomura A and Shirataki Y: Diverse biological activity of a Harvengtens 'Tutu' bulb extracts. In Vivo 25: 381-386, 2011.

6 Patra A, Mitra KA, Biswas S, Gupta DC, Basak A and Barua KA: Carbon-13 NMR spectral studies on some labdane diterpenoids. Magn Reson Chem 16: 75-77, 1981.

7 Srivastava N and Akhila A: Biosynthesis of andrographolide in Andrographis paniculata. Phytochemistry 71: 1298-1304, 2010.

8 Matsuda T, Kuroyanagi M, Sugiyama S, Umehara K, Ueno A and Nishi K: Cell differentiation-inducing diterpenes from Andrographis paniculata Nees. Chem Pharm Bull 42: 12161225, 1994.

9 Suzuki R, Matsuo S, Sakagami H, Okada Y and Shirataki Y: Search of new cytotoxic crude materials against human oral squamous cell carcinoma using NMR metabolomics. Anticancer Res 34: 4117-4120, 2014.
10 Tamura N, Hirano K, Kishino K, Hashimoto K, Amano O, Shimada J and Sakagami H: Analysis of type of cell death induced by topoisomerase inhibitor ( $\mathrm{SN}-38$ ) in human oral squamous cell carcinoma cell lines. Anticancer Res 32: 4823-4832, 2012.

11 Gao $\mathrm{H}$ and Wang J: Andrographolide inhibits multiple myeloma cells by inhibiting the TLR4/NF-kB signaling pathway. Mol Med Rep 13: 1827-1832, 2016.

12 Peng T, Hu M, Wu TT, Zhang C, Chen Z, Huang S and Zhou $\mathrm{XH}$ : Andrographolide suppresses proliferation of nasopharyngeal carcinoma cells via attenuating NF-kB pathway. Biomed Res Int 2015: 735056, 2015.

13 Pratheeshkumar P, Sheeja K and Kuttan G: Andrographolide induces apoptosis in B16F-10 melanoma cells by inhibiting NFкB-mediated BCL-2 activation and modulating p53-induced caspase- 3 gene expression. Immunopharmacol Immunotoxicol 34: 143-151, 2012.

14 Lee KC, Chang HH, Chung YH, and Lee TY: Andrographolide acts as an anti-inflammatory agent in LPS-stimulated RAW264.7 macrophages by inhibiting STAT3-mediated suppression of the NF-kB pathway. J Ethnopharmacol 135: 678-684, 2011.

15 Bundela S, Sharma A and Bisen PS: Potential Compounds for Oral Cancer Treatment: Resveratrol, Nimbolide, Lovastatin, Bortezomib, Vorinostat, Berberine, Pterostilbene, Deguelin, Andrographolide, and Colchicine. PLoS One 10: e0141719, 2015.

Received August 6, 2016

Revised August 31, 2016

Accepted September 5, 2016 